\title{
Educational achievement and fracture risk
}

\author{
E. Clark • J. Tobias
}

Received: 10 September 2009 / Accepted: 29 September 2009 / Published online: 9 December 2009

(C) International Osteoporosis Foundation and National Osteoporosis Foundation 2009

\section{Dear Editors,}

We read with interest the article by Brennan et al. in the September issue of Osteoporosis International describing the association between socio-economic status and osteoporotic fracture in population-based adults [1]. In this systematic review they found a strong association between marital status and fracture, with those who were unmarried, single, divorced or widowed having the highest risk. However, they found conflicting data for an association between educational attainment or level of income and osteoporotic fracture, which they felt was surprising because of the "common assumption that participation in healthier lifestyles increases with higher income and educational attainment'. They suggest some potential explanations for this, but we would like to suggest an alternative.

We carried out a large population-based study of the associations between socio-economic status and bone mass (one of the strongest predictors of osteoporotic fracture) in children [2] and found no overall association between highest educational achievement of the mother and bone mass of the offspring. Instead we found opposing actions of educational achievement on height and weight, which are

A response to this letter can be found at doi 10.1007/s00198-009-

1112-x.

E. Clark $\cdot$ J. Tobias

Academic Rheumatology, University of Bristol,

Bristol, UK

E. Clark $(\bowtie)$

Avon Orthopaedic Centre, Southmead Hospital,

Bristol BS10 5NB, UK

e-mail: emma.clark@Bristol.ac.uk the biggest predictors of bone mass. Those children whose mothers had the highest educational achievement were taller but thinner, and those children whose mothers had minimal formal education were shorter but more obese. We interpreted this as higher educational achievement being associated with longer but more slender bones, whereas lower educational achievement was associated with shorter, wider bones, and as a consequence bone area was the same across the range of educational achievement.

Our work confirms that educational achievement does affect skeletal development, and suggests that the pathway via which educational achievement exerts its effects on bone mass is by opposing actions on height and weight. This may be a further explanation for the conflicting evidence of an association between educational attainment or level of income and osteoporotic fracture in adults. It is likely that the studies found in this comprehensive systematic review did not assess the effects of socioeconomic status on determinants of fracture risk such as bone mass, and they certainly did not assess the effects on determinants of bone mass, particularly height and weight.

Conflicts of interest None

\section{References}

1. Brennan SL, Pasco JA, Urquhart DM, Oldenburg B, Hanna F, Wluka AE (2009) The association between socioeconomic status and osteoporotic fracture in population-based adults: a systematic review. Osteoporos Int 20:1487-1497

2. Clark EM, Ness A, Tobias JH (2005) Social position affects bone mass in childhood through opposing actions on height and weight. J Bone Miner Res 20(12):2082-2089 